\title{
Radical local treatment for stage IV non-small cell lung cancer in older adults: a propensity-score matched analysis of the SEER database
}

\author{
Chenhui Qiu ${ }^{1 \#}$, Shaotang Zhang ${ }^{2 \#}$, Hualiang Jin ${ }^{1}$, Xiaoqin Zhang ${ }^{3}$, Jian Ye ${ }^{1}$ \\ ${ }^{1}$ Departments of Respiratory Medicine, Affiliated Hangzhou First People's Hospital, Zhejiang University School of Medicine, Hangzhou, China; \\ ${ }^{2}$ Departments of Pulmonary Medicine, Fuyang No. 2 Hospital, Fuyang, China; ${ }^{3}$ Department of Respiratory Medicine, Zhejiang Provincial People's \\ Hospital, People's Hospital of Hangzhou Medical College, Hangzhou, China \\ Contributions: (I) Conception and design: C Qiu, S Zhang, J Ye; (II)Administrative support: C Qiu, S Zhang, J Ye; (III) Provision of study materials \\ or patients: X Zhang; (IV) Collection and assembly of data: C Qiu, S Zhang, J Ye; (V) Data analysis and interpretation: C Qiu, S Zhang, J Ye; (VI) \\ Manuscript writing: All authors; (VII) Final approval of manuscript: All authors. \\ "These authors contributed equally to this work. \\ Correspondence to: Jian Ye. Departments of Respiratory Medicine, Affiliated Hangzhou First People's Hospital, Zhejiang University School of \\ Medicine, No. 261, Huansha Road, Hangzhou, China. Email: yejianchi@126.com.
}

Background: Increased incidence of lung cancer in older adults is attributed to increased life expectancy, increased risk for many types of cancer, age-associated comorbidities and physical performance status. Contraindicatory tumor resection is suggested to benefit survival outcomes in stage IV non-small-cell lung cancer (NSCLC). We analyzed the clinical characteristics of older adults $\geq 70$ years old with stage IV NSCLC and investigated whether radical local treatment may benefit this population.

Methods: This retrospective, population-based cohort study analyzed patient data from the USA Surveillance, Epidemiology and End Results (SEER) Program during 2004-2016. Eligible patients were aged $\geq 70$ years and diagnosed with stage IV NSCLC. Primary endpoints were overall survival (OS) and lung-cancer-specific survival (LCSS). Propensity-score matching (PSM) and Cox regression analysis were performed to assess the prognostic role of surgical resection of primary tumor or metastasis.

Results: Among 54,310 stage IV NSCLC older patients, 7.50\% received radical local treatment of the primary tumor or metastasis. PSM resulted in a balanced study population consisting of a treatment group $(n=4,037)$ and a matched no-local-treatment group $(n=15,658)$. After adjusting for confounders, radical local treatment was significantly associated with increased LCSS [hazard ratio (HR): 0.85, 95\% confidence interval (95\% CI): 0.72-1.00] but decreased OS (HR: 1.97, 95\% CI: 1.31-2.97, P=0.0012).

Conclusions: In older adults $\geq 70$ years old with stage IV NSCLC, radical local treatment is associated with increased LCSS but decreased OS. Additional prospective studies are warranted to confirm the benefit of radical local treatment for primary or metastatic NSCLC.

Keywords: Non-small cell lung cancer (NSCLC); metastasis; prognosis; surgery; Surveillance, Epidemiology and End Results Program (SEER Program)

Submitted Dec 13, 2019. Accepted for publication Jul 10, 2020.

doi: $10.21037 /$ tcr-19-2796

View this article at: http://dx.doi.org/10.21037/tcr-19-2796

\section{Introduction}

An increased incidence of lung cancer in older adults is attributed mainly to the increase in human life expectancy and the concomitant increase in the risk of many types of cancer (1). Older patients differ from their younger counterparts by having more comorbidities, including 
more smoking-related comorbidities, and poorer physical performance status and inactivity $(2,3)$. Therefore, this population appears to need focused assessment of their specific lung cancer risk factors and evaluation of appropriate therapies.

The World Health Organization (WHO) reported in 2015 that lung cancer was the fifth leading cause of mortality with 1.7 million deaths worldwide (4). Older adults are affected disproportionately (5), with two-thirds of all new cases occurring in individuals aged 65 years or older (6). Since the twentieth century, the primary therapeutic strategy for patients with stage IV non-small cell lung cancer (NSCLC) has been systemic monotherapy with chemotherapeutic agents such as gemcitabine and vinorelbine, which showed significant benefit in survival that was not surpassed by combination chemotherapy (1). However, aside from results of two phase III trials in Italy showing that therapy with these drugs was more beneficial than supportive care alone in older adults with lung cancer $(7,8)$, evidence of the efficacy of chemotherapy for elderly patients remains limited (2). It has been shown that older adults have higher rates of toxicity when undergoing chemotherapy (9), but evidence is otherwise scarce. Treatment also may be complicated by multiple morbidities that are common among older adults (10). Also, compromised immune systems and comorbidities make older adults a "special population", and they are not always accepted in clinical trials; the classic endpoints also are not valid in geriatric populations because causes of death cannot always be ascribed precisely as disease progression, toxicity, or comorbid conditions (11).

More recently, radical approaches have been suggested for select patients with stage IV disease, including surgery for primary and metastatic disease (12-14). However, we do not know whether patients with more advanced age may receive the potential benefits of radical local treatments as readily as younger patients. Although some older adults are reported to tolerate surgical treatment deemed appropriate for their tumor stage (9), treatment strategies still appear to differ considerably between age groups (3). While curative-intent treatment and survival have increased for all NSCLC patients over time, the increase is less substantial among older adult patients (3). Several recent studies have documented the potential benefits of surgery on survival for patients with metastatic NSCLC (12-14), and all reported survival benefits and an apparent beneficial role for surgery. Although these studies were not focused exclusively on elderly NSCLC patients, one study reported a median age of $65-70$ years, and the authors suggested that definitive conclusions cannot be made about the role of surgery in older patients with metastatic disease until more information is available about systemic therapies and comorbidities (14).

Two studies that focused specifically on older adult patients with NSCLC both emphasized that comorbid conditions are often the reason for exclusion from surgical resection on the basis of tumor stage, and that understanding predictive factors may help in the selection of older patients who are likely to benefit from surgical treatment $(15,16)$. Therefore, the purpose of the present study was to analyze the clinical characteristics of older adults with stage IV NSCLC and to determine whether radical local treatment may benefit this patient population. We present the following article in accordance with the STROBE reporting checklist (available at http://dx.doi. org/10.21037/tcr-19-2796).

\section{Methods}

\section{Data source}

This study extracted patient data from the Surveillance, Epidemiology and End Results (SEER) Program (www.seer. cancer.gov) Research Data (2004-2016), National Cancer Institute (NCI), Division of Cancer Control and Population Sciences (DCCPS), Surveillance Research Program (SRP), Surveillance Systems Branch. The SEER program is a national collaboration of health information agencies in the United States that collects data from cancer registries on cancer incidence and survival from 18 geographic areas around the USA, representing about $30 \%$ of the USA population (17). The SEER database contains information from approximately three million patients with cancer from a variety of geographic regions in the USA. The SEER program has authorized access to the database by research scientists who may request permission from the NCI (18).

\section{Study design and ethical considerations}

This is retrospective, population-based cohort study. All SEER data obtained from electronic hospital medical information systems are de-identified by the SEER Program and data analysis for research purposes does not require approval of the Internal Review Board or informed consent by participating subjects. The study was conducted in accordance with the Declaration of Helsinki (as revised in 2013). 


\section{Study population}

Patients considered eligible for inclusion were those aged 70 years and older who were diagnosed with any of the following histological subtypes of NSCLC: NSCLC not otherwise specified, adenocarcinoma, squamous cell carcinoma or large cell carcinoma or any of their variants. All codes and rules in the present study followed guidelines established by the SEER program. Patient data were extracted from the SEER-18 cancer registry based on the International Classification of Diseases for Oncology, Third Revision (ICD-O-3) codes (8010/3, 8012/3, 8020/3, $8046 / 3,8050-8052 / 3,8070-8078 / 3,8140 / 3,8141 / 3$, $8143 / 3,8147 / 3,8250-8255 / 3,8260 / 3,8310 / 3,8430 / 3$, $8480 / 3,8481 / 3,8490 / 3,8560 / 3$, and $8570-8575 / 3)$ and topographical codes C34.0-C34.9 (bronchus and lung). The primary cohort included only patients with stage IV disease at presentation, with definitive $\mathrm{T}$ and $\mathrm{N}$ stage, and with only one primary site. Patients for whom it was unknown if surgery was performed or not, or with missing information regarding race/ethnicity and follow-up time, were excluded from the primary cohort.

\section{Endpoints}

Patient outcomes were obtained from the SEER database until December 31, 2013. The primary endpoints were overall survival (OS) which was calculated from the date of diagnosis to the date of death due to any cause, and lungcancer-specific survival (LCSS), which was calculated from diagnosis until cause-specific death from lung cancer.

\section{Study variables}

Independent variables evaluated for each case included patients' demographic data (age at diagnosis, sex, race/ ethnicity) and clinical characteristics (tumor histologic subtypes, characteristics of metastasis, $\mathrm{T}$ and $\mathrm{N}$ stage), whether or not they received radical local treatment (defined as resection of primary tumor or metastasis), and whether or not they received radiation therapy as the first course of treatment. Information about chemotherapy received and adjuvant treatment after the first course were not recorded in the SEER database and therefore were not included or analyzed.

\section{Propensity-score matching (PSM)}

Selection bias is always a concern especially in retrospective study design. Because the included cohort was nonrandomized, unbalanced baseline characteristics may exist and selection bias may undermine estimation of the treatment effect. Therefore, a PSM algorithm was applied to minimize the influence of possible confounding by indication. The propensity score is defined in this analysis as the probability of being assigned to surgery for the primary tumor or metastatic disease. In the calculation of propensity scores, the logistic regression model was used considering the following baseline characteristics: age, sex, race, $\mathrm{T}$ stage, $\mathrm{N}$ stage, site of metastases, histological subtypes, characteristics of metastasis and whether or not radiation was received. Patients who received surgical treatment for the primary tumor or metastases were matched with patients who received no surgical treatment with an algorithm of nearestneighbor 1:4 matching. This approach has been validated and applied in previously published studies (14,19-21).

\section{Statistical analysis}

Patients' demographic and clinical characteristics are summarized as $\mathrm{n}$ (\%) for total patients, and OS and lungcancer-specific-survival (LCSS) are summarized as n (\%) for patients' given demographic and clinical characteristics. Univariate and multivariate Cox regression analyses were performed to estimate the effects of surgical treatment on OS and LCSS. Variables with significant associations $(\mathrm{P}<0.05)$ in univariate analysis were placed into multivariate analysis. Results are presented as hazard ratios (HRs) with corresponding $95 \%$ confidence intervals (95\% CIs) and P values. OS and LCSS were both graphed in Kaplan-Meier curves for total patients and for given surgery types along with log-rank test. Median survival times with $95 \%$ CI of median time were summarized accordingly. All statistical assessments were two-tailed and considered significant at $\mathrm{P}<0.05$. Data were analyzed using SAS 9.0 (SAS Institute Inc., Cary, NC, USA).

\section{Results}

\section{Basic characteristics}

Between January 1, 2004 and December 31, 2016, the SEER database collected data on 6,024,624 patients. After the inclusion and exclusion criteria were applied, the final study cohort consisted of 54,310 patients. A flow diagram of the selection process is presented in Figure 1.

Overall, 4,075 patients $(7.50 \%)$ received radical local 


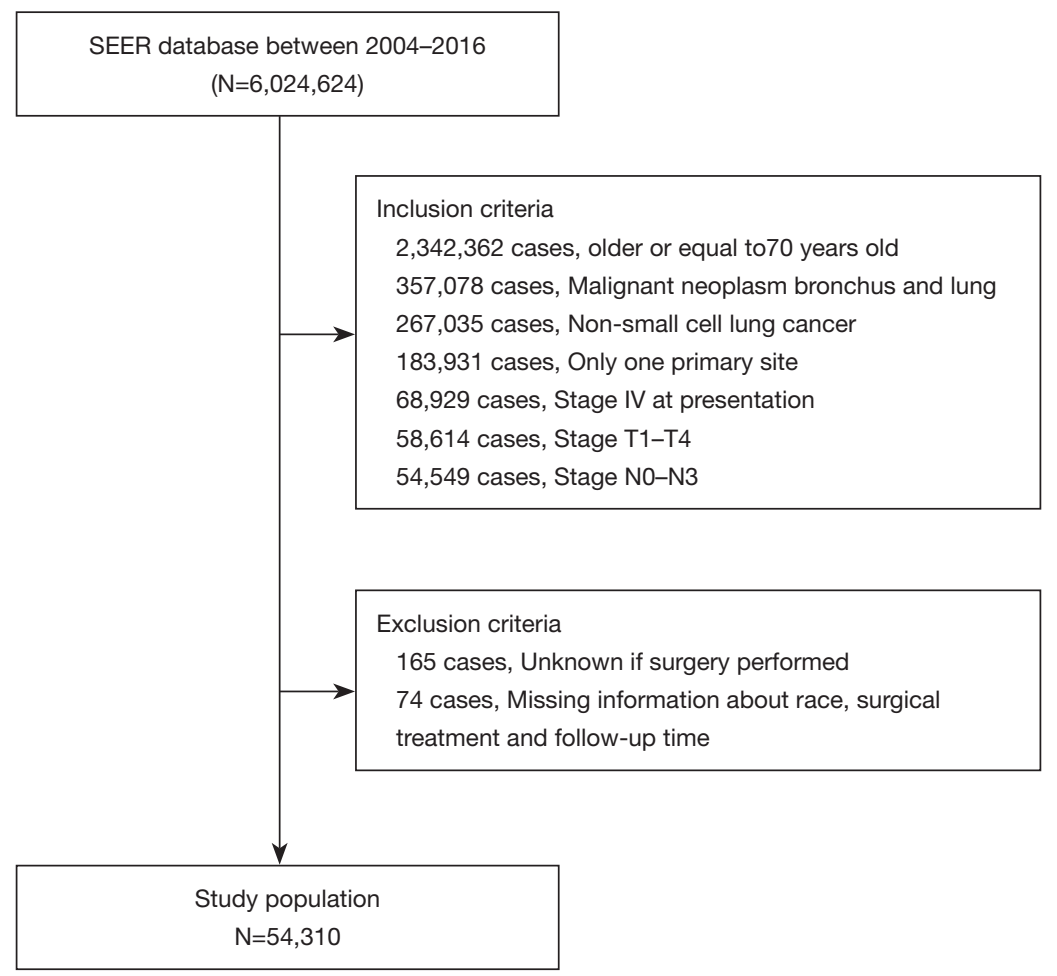

Figure 1 Flow chart of selection population.

treatment, and 50,235 patients $(92.50 \%)$ did not. Patients' baseline characteristics are summarized in Table 1. Patients receiving radical local treatment were younger $(\mathrm{P}<0.0001)$, more likely to be white $(\mathrm{P}=0.0045)$, with histology of adenocarcinoma variants $(\mathrm{P}<0.0001)$, with metastasis to distant organs $(\mathrm{P}<0.0001)$, of $\mathrm{T} 1-\mathrm{T} 2$ stage $(\mathrm{P}<0.001)$, node positive $(\mathrm{P}<0.0001)$ and did not receive radiation $(\mathrm{P}<0.0001)$ (Table 1).

\section{Survival outcomes before PSM}

Kaplan-Meier curves of OS and LCSS according to whether or not radical local treatment was performed in the unmatched population are shown in Figure $2 A, B$. For the whole population, a statistically significant difference was found in OS and LCSS favoring the local treatment group (OS, $\mathrm{P}=0.0009$; LCSS, $\mathrm{P}<0.0001$ ) (Figure 2).

\section{Survival outcomes after PSM}

The propensity matching process resulted in a balanced study population consisting of a radical local treatment group $(n=4,037)$ and a matched no-local-treatment group $(\mathrm{n}=15,658)$. Table 2 shows the distribution of study variables before and after matching. No significant differences were found regarding age, sex, race/ethnicity, $\mathrm{T}$ stage, $\mathrm{N}$ stage and histological subtype between the two groups after PSM (Table 2). Kaplan-Meier curves of OS and LCSS after PSM according to whether or not radical local treatment was performed are shown in Figure $3 A, B$.

The results of univariate and multivariate Cox regression analyses for OS in the matched population are shown in Table 3. In univariate analysis, radical local treatment was associated with increased OS (HR: 0.85, 95\% CI: 0.76-0.95, $\mathrm{P}=0.0054)$. However, after adjustment in multivariate analysis, radical local treatment was significantly associated with decreased OS (HR: 1.97, 95\% CI: 1.31-2.97, $\mathrm{P}=0.0012$ ). The results also indicated that race/ethnicity, histological subtype, characteristics of metastasis, T stage, surgical extension of primary tumor, surgery for metastatic disease and radiation therapy were independent prognostic factors for OS (All $\mathrm{P}<0.05$ ) (Table 3).

Table 4 shows the results of univariate and multivariate Cox regression analyses of prognostic factors for LCSS in the matched population. In univariate analysis, radical local treatment was associated with increased LCSS (HR: 0.60, 
Table 1 Baseline characteristics of stage IV NSCLC patients included in the analysis

\begin{tabular}{|c|c|c|}
\hline Characteristics & Radical local treatment (surgery to primary tumor or metastases), $\mathrm{n}(\%)$ & No radical local treatment, $\mathrm{n}(\%)$ \\
\hline \multicolumn{3}{|l|}{ Age, years } \\
\hline $70-84$ & $3,844(94.33)$ & $43,448(86.49)$ \\
\hline$\geq 85$ & $231(5.67)$ & $6,787(13.51)$ \\
\hline Female & $1,977(48.52)$ & $23,714(47.21)$ \\
\hline Male & $2,098(51.48)$ & $26,521(52.79)$ \\
\hline \multicolumn{3}{|l|}{ Race } \\
\hline White & $3,423(84.00)$ & $41,198(82.01)$ \\
\hline \multicolumn{3}{|l|}{ Histological subtype } \\
\hline NSCLC, not otherwise specified & $577(14.16)$ & $14,168(28.20)$ \\
\hline Squamous cell carcinoma variants & $774(18.99)$ & $10,810(21.52)$ \\
\hline Large cell carcinoma & $152(3.73)$ & $1,314(2.62)$ \\
\hline Adenocarcinoma variants & $2,572(63.12)$ & $23,943(47.66)$ \\
\hline \multicolumn{3}{|l|}{ Characteristics of metastasis } \\
\hline Distant organ metastases & $1,914(46.97)$ & $25,246(50.26)$ \\
\hline Distant lymph node metastases & $113(2.77)$ & $985(1.96)$ \\
\hline T3-T4 & $1,977(48.52)$ & $31,767(63.24)$ \\
\hline \multicolumn{3}{|l|}{$\mathrm{N}$ stage } \\
\hline Node positive & $2,236(54.87)$ & $36,393(72.45)$ \\
\hline Node negative & $1,839(45.13)$ & $13,842(27.55)$ \\
\hline \multicolumn{3}{|l|}{ Surgical extent of primary tumor } \\
\hline None & $1,968(48.29)$ & - \\
\hline Local tumor destruction & $101(2.48)$ & - \\
\hline Sublobular resection & 949 (23.29) & - \\
\hline Lobectomy & $921(22.60)$ & - \\
\hline Pneumonectomy & $87(2.13)$ & - \\
\hline Surgery, no otherwise specified & $49(1.20)$ & - \\
\hline
\end{tabular}

Table 1 (continued) 
Table 1 (continued)

\begin{tabular}{lcc}
\hline Characteristics & Radical local treatment (surgery to primary tumor or metastases), $\mathrm{n}(\%)$ & No radical local treatment, $\mathrm{n}(\%)$ \\
\hline Surgery to metastases & $1,857(45.57)$ & - \\
None & $2,218(54.43)$ & - \\
Yes & & $31,553(63.23)$ \\
Radiation & $2,411(59.72)$ & $18,351(36.77)$ \\
None & $1,626(40.28)$ & 18,37 \\
Yes &
\end{tabular}

NSCLC, non-small cell lung cancer.

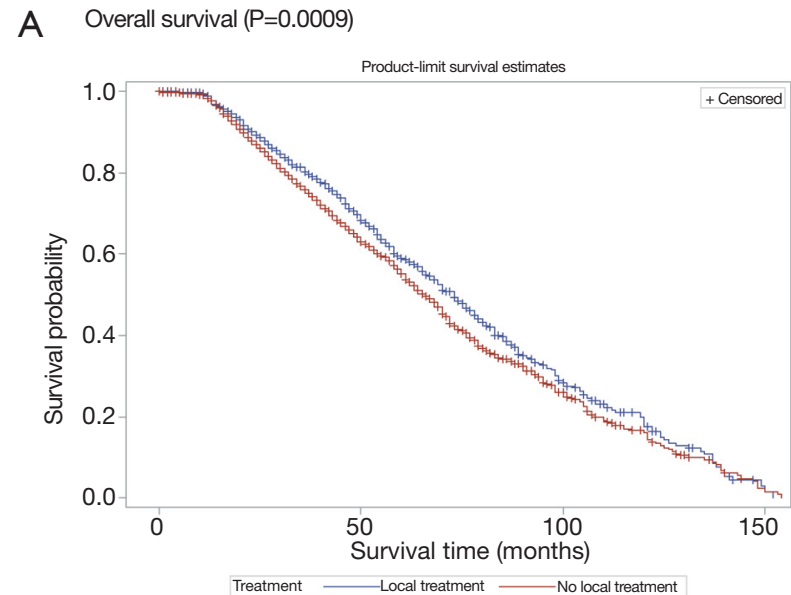

B Lung cancer-specific survival $(P<0.0001)$

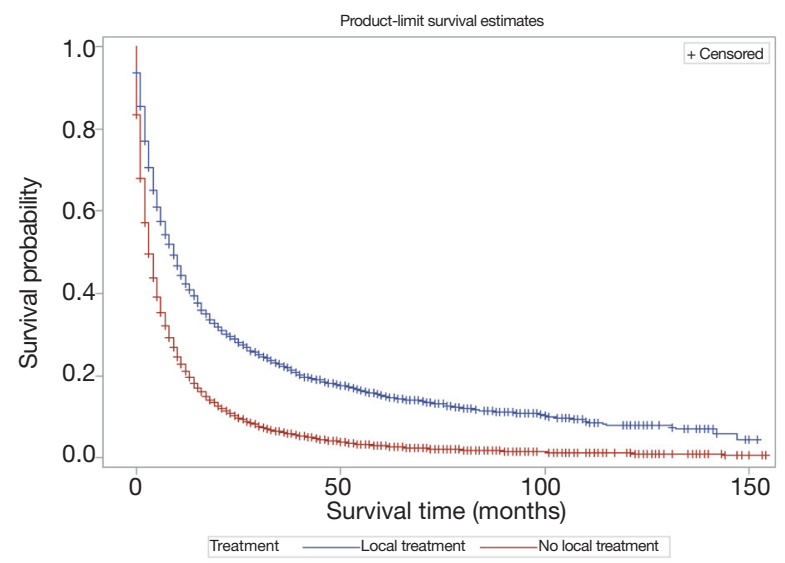

Figure 2 Kaplan-Meier curve of (A) OS and (B) LCSS between radical local treatment or not (before PSM). OS, overall survival; LCSS, lung-cancer-specific survival; PSM, propensity-score matching.
95\% CI: 0.58-0.63, P<0.0001). After adjustments, radical local treatment remained significantly associated with increased LCSS (HR: 0.85, 95\% CI: 0.72-1.00, $\mathrm{P}=0.0458$ ). Sex, age, race/ethnicity, histological subtype, characteristics of metastasis, $\mathrm{T}$ stage, $\mathrm{N}$ stage, surgical extent of primary tumor and radiation were the other significant prognostic factors for LCSS (All $\mathrm{P}<0.05)$ (Table 4).

\section{Discussion}

In the present study, we evaluated the prognostic role of radical local treatment in adults aged 70 years and older diagnosed with stage IV NSCLC, using PSM to balance the preoperative patient characteristics in the treatment and comparison groups to obtain less biased estimates. After adjusting for all confounders, radical local treatment was shown to be significantly associated with increased LCSS, and a $15 \%$ risk reduction of lung-cancer-specific death. In contrast, however, radical local treatment was associated with decreased OS in this populations, that is, increased risk for overall morality.

Several recent studies have examined the potential benefits of surgical resection specifically in older adult populations with NSCLC. Okamoto et al. (15) attempted to clarify the surgical management of NSCLC patients over age 75 years-a group among whom comorbid conditions often lead to intraoperative or postoperative complications. In 44 patients receiving partial resection or lobectomy, no significant differences were found in variables between the two groups but a significant difference was seen in OS in all but stage I patients, and no difference was seen between groups in disease-free survival. The authors noted less surgical risk in sub-lobar resection and generally better 
Table 2 Patients' demographic and clinical characteristics before and after PSM

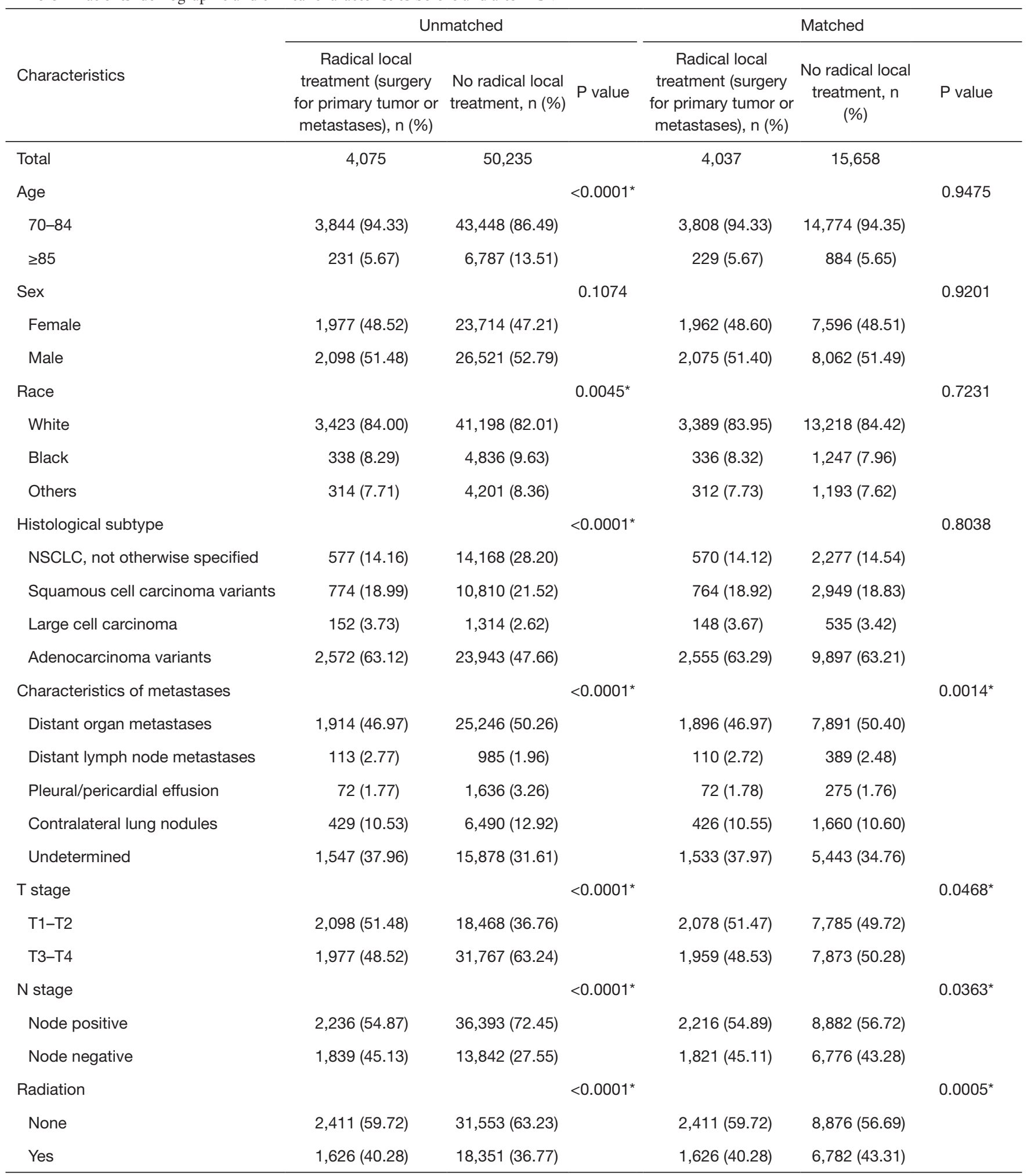

Significant values are in *. PSM, propensity-score matching; NSCLC, non-small cell lung cancer. 
A Overall survival $(\mathrm{P}=0.0051)$

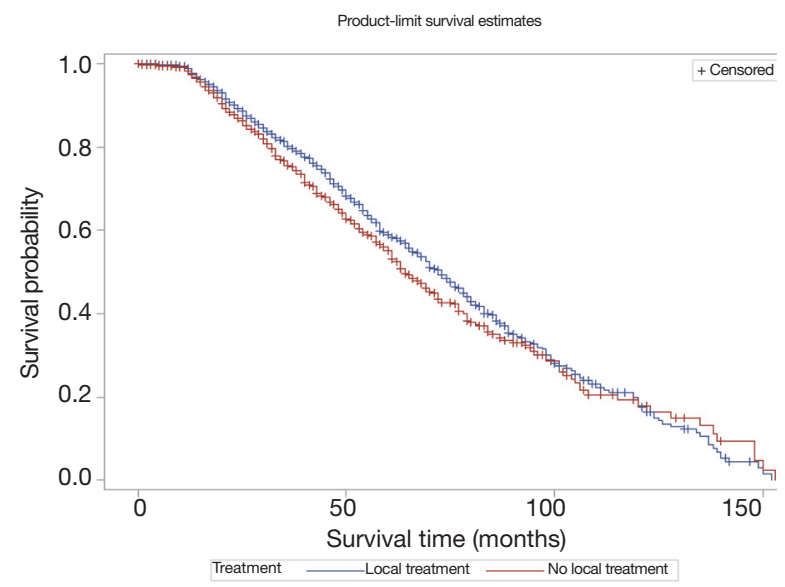

B

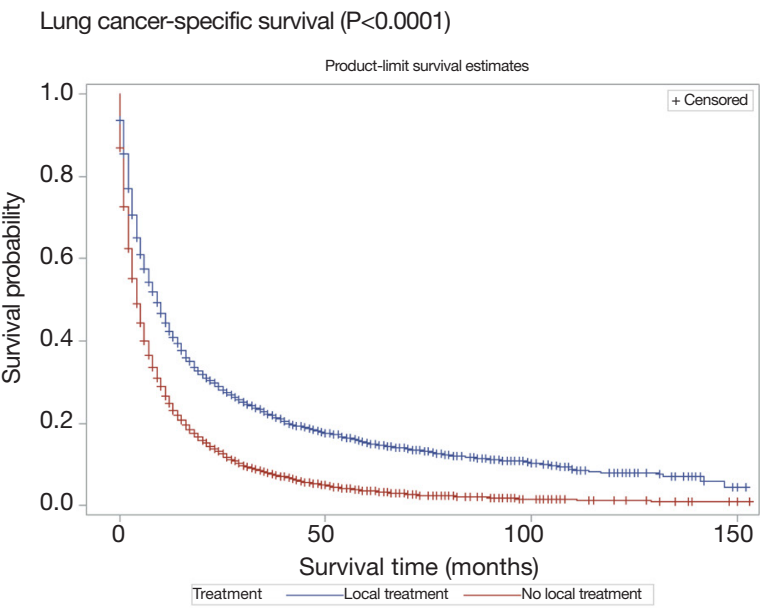

Figure 3 Kaplan-Meier curve of (A) OS and (B) LCSS between local treatment or not (after PSM). OS, overall survival; LCSS, lung-cancer-specific survival; PSM, propensity-score matching.

outcomes. Stage was important in another study in which those with stages I or II had a median OS of 5.6 compared to 3.5 years in those receiving stereotactic body radiation therapy (SBRT) (22). In adult patients, but not necessarily elderly, with stage IV NSCLC, surgery for primary and metastatic sites were predictive of OS, and survival benefits were not found in metastasis surgery for those with squamous or large cell carcinoma or N1 staging; and the maximum benefit was shown in NSCLC patients receiving primary site surgeries with stage N0 (23). Another study of aggressive surgical strategy for stage IV NSCLC showed a 5 -year OS of $23.3 \%$ and a disease-free survival of $15.8 \%$; those authors suggested that surgical treatment of stage
IV disease can extend life only if the patients can tolerate surgery, which may again exclude elderly patients (24). Patients with stage IV NSCLC are considered incurable and most often receive best supportive care with the intent of palliation rather than cure; but surgical intervention for NSCLC patients remains controversial between studies and authors. Kwint et al. (25) investigated OS and progressionfree survival (PFS) in NSCLC patients with mean age 60 years who received radical treatment consisting of systemic treatment, radical radiotherapy, and intrathoracic surgical resection or radiofrequency ablation. In that study, favorable OS and PFS were found in patients with good performance status, which may point to a key factor in determining potential survival benefit for older adult patients undergoing surgical or other radical treatment for lung cancer.

In a narrative review by David et al. (12), it was suggested that future studies should focus on possibly including surgery as part of the therapeutic strategy, in addition to systemic therapies for appropriately selected stage IV NSCLC patients. A previous study that also used data of NSCLC patients from the SEER database found, as we did, that all types of surgery (primary site, metastasis site, or both) had significantly better OS than no surgery, even in stage IV patients (26). The deciding factor in all studies seems to suggest patient selection, considering age, comorbidities, and specific sites of metastases (liver and bone had poorer survival statistics than adrenal or nervous system metastases); stage also appears to be a determinate of the aggressiveness of surgery, with complete resection contributing to better survival than partial (27). In contrast, Yang et al. (13) conducted a population-based study using a nationwide database and evaluated prognostic factors for survival of specific M1 diseases, concluding that surgery does not appear to compromise outcomes as compared to non-surgical therapies. Also, results of multivariate analysis reported by Abdel-Rahman (14) showed that combined surgery of primary tumor and metastatic disease did not benefit OS in oligometastatic NSCLC. Similarly, results of the present study show that although surgery increases cancer-specific survival, it decreases OS in the same population. Taken together, these inconsistent findings in our study and the evidence in the literature do not allow a definitive conclusion to be made about whether radical local treatment benefits survival, and which patient subgroup may benefit from surgery.

Nearly all authors examining surgery for NSCLC, recognized the need for additional studies on chemotherapy among the older adult patient population due to the 
Table 3 Prognostic factors for OS in the matched population

\begin{tabular}{|c|c|c|c|c|c|c|}
\hline Prognostic factors & \multicolumn{3}{|c|}{ Univariate } & \multicolumn{3}{|c|}{ Multivariate } \\
\hline \multicolumn{7}{|l|}{ Radical local treatment } \\
\hline None & \multicolumn{3}{|c|}{ Reference } & \multicolumn{3}{|c|}{ Reference } \\
\hline Yes & -0.16 & $0.85(0.76,0.95)^{*}$ & $0.0054^{*}$ & 0.68 & $1.97(1.31,2.97)^{\star}$ & $0.0012^{*}$ \\
\hline Male & \multicolumn{3}{|c|}{ Reference } & \multicolumn{3}{|c|}{ Reference } \\
\hline Female & 0.07 & $1.07(0.96,1.20)$ & 0.229 & 0.07 & $1.08(0.96,1.21)$ & 0.2013 \\
\hline \multicolumn{7}{|l|}{ Age } \\
\hline $70-84$ & \multicolumn{3}{|c|}{ Reference } & \multicolumn{3}{|c|}{ Reference } \\
\hline White & \multicolumn{3}{|c|}{ Reference } & \multicolumn{3}{|c|}{ Reference } \\
\hline Black & 0.11 & $1.11(0.90,1.37)$ & 0.3135 & 0.12 & $1.12(0.91,1.38)$ & 0.2767 \\
\hline Others & 0.33 & $1.4(1.19,1.64)^{*}$ & $<0.0001^{*}$ & 0.3 & $1.35(1.15,1.60)^{*}$ & $0.0003^{\star}$ \\
\hline \multicolumn{7}{|l|}{ Histological subtype } \\
\hline Adenocarcinoma variants & \multicolumn{3}{|c|}{ Reference } & \multicolumn{3}{|c|}{ Reference } \\
\hline NSCLC, not otherwise specified & -0.52 & $0.59(0.47,0.76)^{\star}$ & $<0.0001^{*}$ & -0.51 & $0.6(0.47,0.76)$ & $<0.0001^{*}$ \\
\hline Squamous cell carcinoma variants & -0.15 & $0.86(0.74,1.00)$ & 0.0536 & -0.15 & $0.86(0.73,1)$ & 0.0552 \\
\hline Large cell carcinoma & -0.87 & $0.42(0.24,0.73)^{\star}$ & $0.0019^{\star}$ & -0.89 & $0.41(0.24,0.71)$ & $0.0016^{\star}$ \\
\hline Undetermined & 0.32 & $1.38(1.21,1.58)^{\star}$ & $<0.0001^{*}$ & 0.4 & $1.49(1.30,1.71)^{*}$ & $<0.0001^{*}$ \\
\hline \multicolumn{7}{|l|}{ T stage } \\
\hline $\mathrm{T} 1-\mathrm{T} 2$ & \multicolumn{3}{|c|}{ Reference } & \multicolumn{3}{|c|}{ Reference } \\
\hline T3-T4 & -0.12 & $0.89(0.79,1.00)^{\star}$ & $0.0436^{\star}$ & -0.19 & $0.83(0.74,0.93)^{*}$ & $0.0022^{*}$ \\
\hline \multicolumn{7}{|l|}{$\mathrm{N}$ stage } \\
\hline Node positive & \multicolumn{3}{|c|}{ Reference } & \multicolumn{3}{|c|}{ Reference } \\
\hline Node negative & -0.13 & $0.87(0.78,0.98)^{\star}$ & $0.0202^{*}$ & -0.11 & $0.9(0.80,1.01)$ & 0.068 \\
\hline
\end{tabular}

Table 3 (continued) 
Table 3 (continued)

\begin{tabular}{|c|c|c|c|c|c|c|}
\hline Prognostic factors & \multicolumn{3}{|c|}{ Univariate } & \multicolumn{3}{|c|}{ Multivariate } \\
\hline \multicolumn{7}{|l|}{ Surgical extent of primary tumor } \\
\hline None & & Reference & & & Reference & \\
\hline Local tumor destruction & -0.7 & $0.5(0.23,1.05)$ & 0.0652 & -1.3 & $0.27(0.12,0.63)^{*}$ & $0.0022^{*}$ \\
\hline Lobectomy & -0.25 & $0.78(0.67,0.91)^{*}$ & $0.0013^{*}$ & -0.91 & $0.4(0.27,0.61)^{\star}$ & $<0.0001^{*}$ \\
\hline Pneumonectomy & -0.1 & $0.91(0.56,1.47)$ & 0.6925 & -0.79 & $0.45(0.24,0.86)^{*}$ & $0.0154^{*}$ \\
\hline Surgery, no otherwise specified & 0.06 & $1.06(0.44,2.55)$ & 0.8992 & -0.76 & $0.47(0.18,1.22)$ & 0.1188 \\
\hline \multicolumn{7}{|l|}{ Surgery to metastases } \\
\hline \multicolumn{7}{|l|}{ Radiation } \\
\hline None & & Reference & & & Reference & \\
\hline Yes & 0.15 & $1.16(1.04,1.30)^{*}$ & $0.0105^{\star}$ & 0.15 & $1.16(1.02,1.31)^{*}$ & $0.0239^{*}$ \\
\hline
\end{tabular}

Significant values are in *. OS, overall survival; HR, hazard ratio; $95 \% \mathrm{CI}, 95 \%$ confidence interval; NSCLC, non-small-cell lung cancer.

absence of elderly patients in studies that evaluated systemic therapy for NSCLC. Nevertheless, because systemic therapy remains an important part of the picture in treating NSCLC in older adults, we must also mention briefly the recent advances such as pembrolizumab as firstline therapy for metastatic NSCLC. After conducting an earlier study of the potential benefits of pembrolizumab, a monoclonal immunotherapy that blocks interaction between programmed death-1 and programmed-deathligands 1 and 2 (28), the author recently published a review study examining trials evaluating pembrolizumab as first-line therapy for NSCLC; both OS and PFS were significantly improved and adverse outcomes were fewer than with platinum-based chemotherapy (29). In discussing both studies, the author suggested that both the antitumor effects of pembrolizumab and patient selection are jointly responsible for the positive outcomes. A phase III trial (KEYNOTE trial) (30) found that pembrolizumab improved or maintained health-related quality of life in NSCLC patients compared to chemotherapy and may represent the new standard of care for advanced NSCLC patients who express programmed death ligand-1. Although this issue is beyond the scope of the present study, future studies are needed to take such new systemic therapies into account.

\section{Strengths and limitations}

Results of the present study were strengthened by using the large and comprehensive SEER database with its vast number of patients representing almost one-third of the US population. To the best of our knowledge, this study is among the first to evaluate the prognostic role of radical local treatment in stage IV NSCLC in patients aged 70 years and older.

This study also has several limitations. First, it is a retrospective study and the evidence derived may be of lower methodological quality than that from randomized trials. Also, since only the patients who had complete information were included in the analysis, selection bias may possibly exist. The SEER database has under-reported the information and details of chemotherapy and radiotherapy and did not include data of concomitant systemic therapy and interventions after the first course of treatment, either of which may have influenced our results if included. The SEER data also lacked important information about the physical performance status and comorbidities of the included patients, so these data could not be included in the analyses. Also, although important, data of postoperative complications, quality of life and cost-effectiveness of the radical local treatment were not included in the database 
Table 4 Prognostic factors for LCSS in the matched population

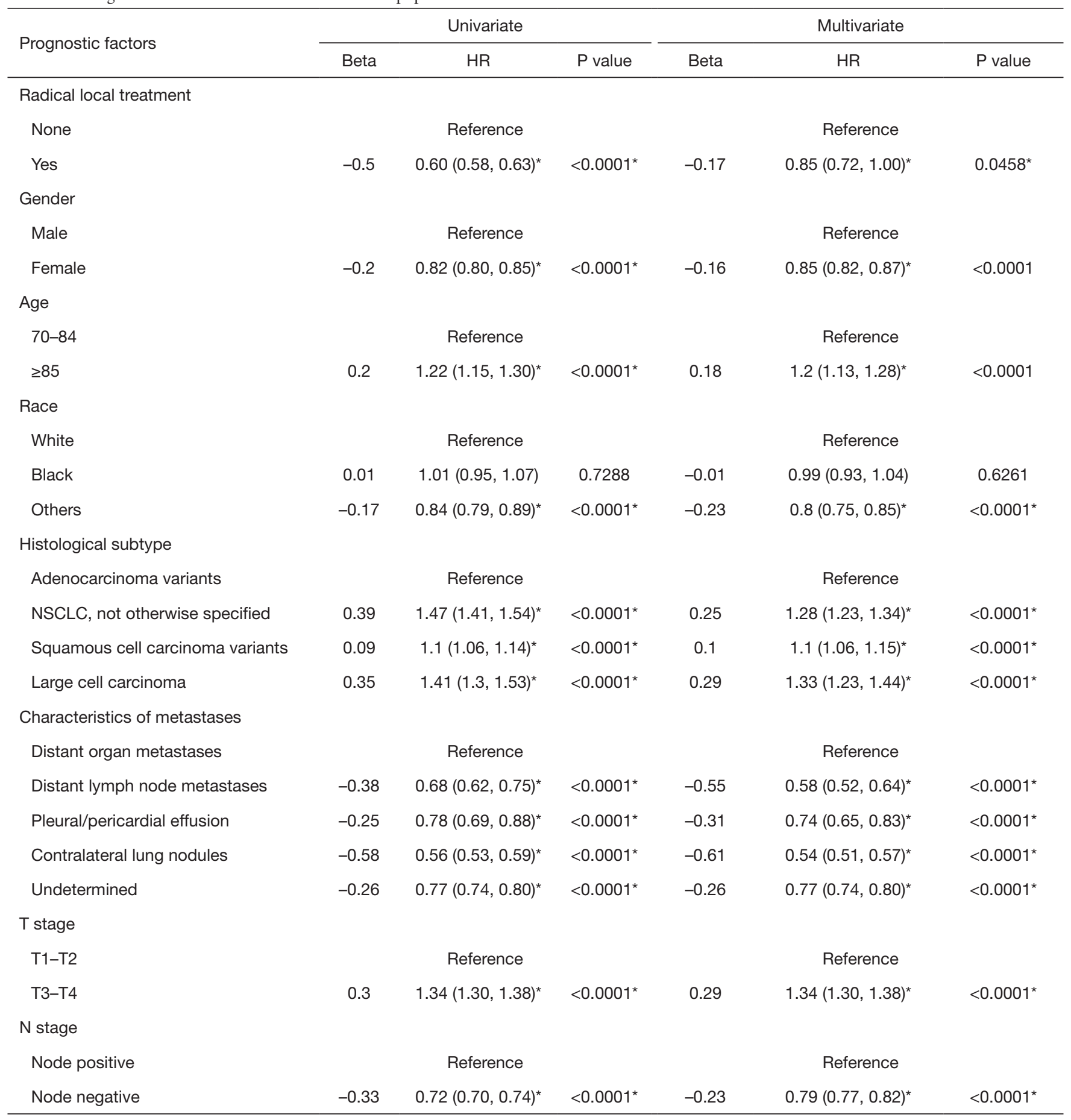

Table 4 (continued) 
Table 4 (continued)

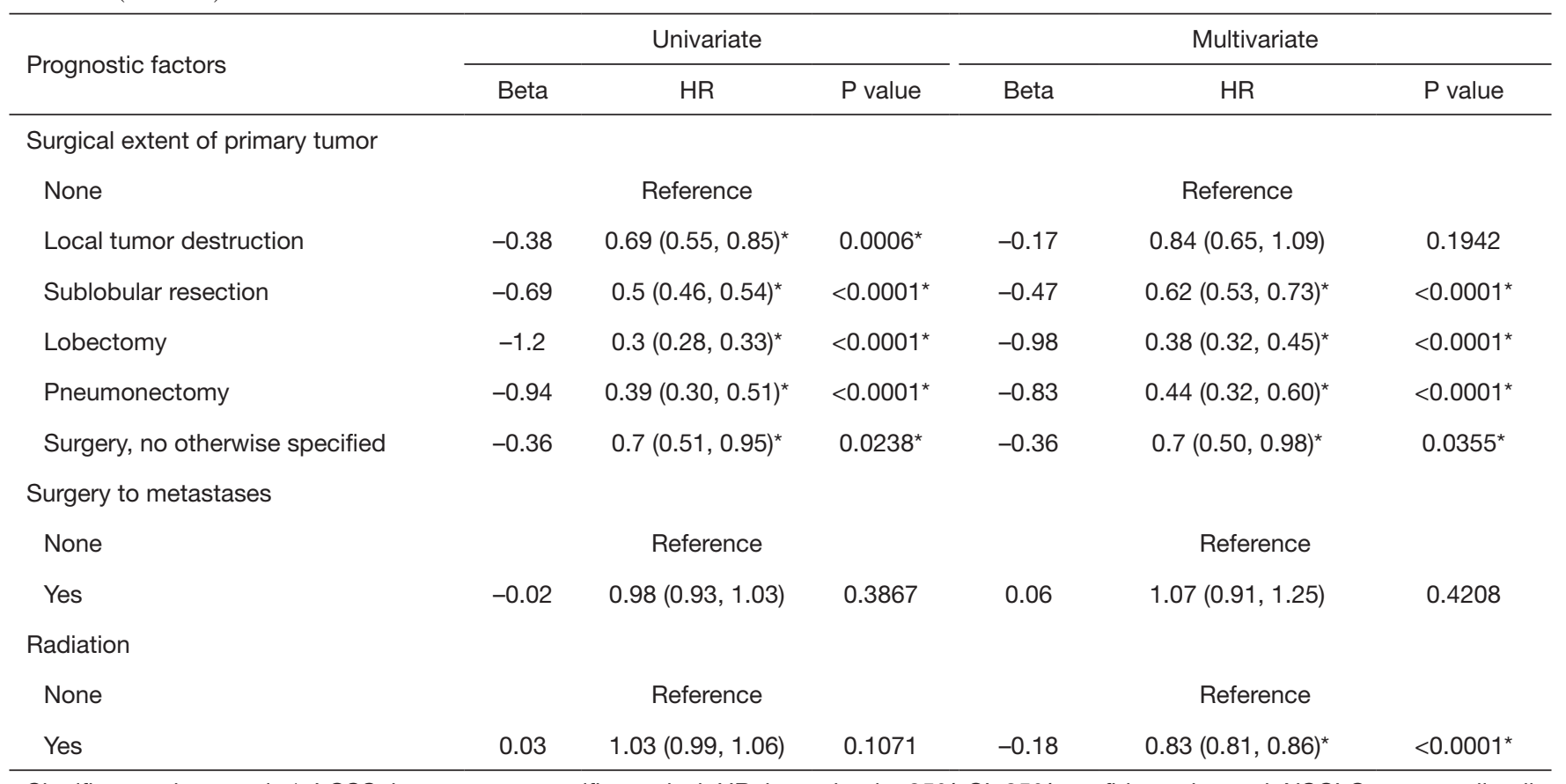

Significant values are in *. LCSS, lung-cancer-specific survival; HR, hazard ratio; 95\% CI, 95\% confidence interval; NSCLC, non-small-cell lung cancer.

and therefore could not be investigated. Data of exact distribution of metastases also was unknown. Prospective studies are still needed to confirm our findings, considering and adjusting for comorbidities, performance status and systemic therapies.

\section{Conclusions}

In stage IV NSCLC in patients aged 70 years and older, radical local treatment is associated with increased LCSS but OS is decreased. The inconsistent finding in our study and others in the literature hinder a definitive conclusion on the role of surgery as part of the therapeutic strategy for this population. Further well-designed prospective studies are warranted with careful adjustment of comorbidities, patients' performance status and systemic therapies.

\section{Acknowledgments}

The authors wish to acknowledge the SEER Program tumor registries for efforts in the construction of the SEER database. The interpretation and reporting of these data are the sole responsibility of the authors. We also wish to acknowledge the medical writing and editorial assistance provided by MedCom Asia Inc.

Funding: This study was supported by the Medical Science and Technology Project Foundation of Zhejiang Province (project number: 2015KYB288, 2019KY490) and by the Research Project of Public Welfare Technology Application of Zhejiang Province Science and Technology Department (project number: 2017C33124).

\section{Footnote}

Reporting Checklist: The authors have completed the STROBE reporting checklist. Available at http://dx.doi. org/10.21037/tcr-19-2796

Conflicts of Interest: All authors have completed the ICMJE uniform disclosure form (available at http://dx.doi. org/10.21037/tcr-19-2796). The authors have no conflicts of interest to declare.

Ethical Statement: The authors are accountable for all aspects of the work in ensuring that questions related to the accuracy or integrity of any part of the work are appropriately investigated and resolved. This is retrospective, population-based cohort study. All SEER 
data obtained from electronic hospital medical information systems are de-identified by the SEER Program and data analysis for research purposes does not require approval of the Internal Review Board or informed consent by participating subjects.

Open Access Statement: This is an Open Access article distributed in accordance with the Creative Commons Attribution-NonCommercial-NoDerivs 4.0 International License (CC BY-NC-ND 4.0), which permits the noncommercial replication and distribution of the article with the strict proviso that no changes or edits are made and the original work is properly cited (including links to both the formal publication through the relevant DOI and the license). See: https://creativecommons.org/licenses/by-nc-nd/4.0/.

\section{References}

1. Quoix E. Therapeutic options in older patients with metastatic non-small cell lung cancer. Ther Adv Med Oncol 2012;4:247-54.

2. Takayuki N, Keiko T, Junji U, et al. Advanced nonsmall-cell lung cancer in elderly patients: patient features and therapeutic management. Biomed Res Int 2018;2018:8202971.

3. Driessen EJ, Aarts MJ, Bootsma GP, et al. Trends in treatment and relative survival among Non-Small Cell Lung Cancer patients in the Netherlands (1990-2014): Disparities between younger and older patients. Lung Cancer 2017;108:198-204.

4. World Health Organization. Top 10 Causes of Death. Available online: https://www.who.int/news-room/factsheets/detail/the-top-10-causes-of-death (Accessed April 10, 2019).

5. Gajra A, Akbar SA, Din NU. Management of lung cancer in the elderly. Clin Geriatr Med 2016;32:81-95.

6. Seigel R, Jamal A. Cancer facts and figures. Available online: http://www.cancer.org/acs/groups/content/@ editorial/documents/document/acspc-044552.pdf (Accessed April 18, 2019).

7. Effects of vinorelbine on quality of life and survival of elderly patients with advanced non-small-cell lung cancer. The Elderly Lung Cancer Vinorelbine Italian Study Group. J Natl Cancer Inst 1999;91:66-72.

8. Gridelli C, Perrone F, Gallo C, et al. Chemotherapy for elderly patients with advanced non-small-cell lung cancer: the Multicenter Italian Lung Cancer in the Elderly Study (MILES) phase III randomized trial. J Natl Cancer Inst
2003;95:362-72.

9. Barta JA, Zinner RG, Unger M. Lung cancer in the older patient. Clin Geriatr Med 2017;33:563-77.

10. Leduc C, Antoni D, Charloux A, et al. Comorbidities in the management of patients with lung cancer. Eur Respir J 2017;49:1601721.

11. Blanco R, Maestu I, de la Torre MG, et al. A review of the management of elderly patients with non-small-cell lung cancer. Ann Oncol 2015; 26:451-63.

12. David EA, Clark JM, Cooke DT, et al. The role of thoracic surgery in the therapeutic management of metastatic nonsmall cell lung cancer. J Thorac Oncol 2017;12:1636-45.

13. Yang CJ, Gu L, Shah SA, et al. Long-term outcomes of surgical resection for stage IV non-small-cell lung cancer: a national analysis. Lung Cancer 2018;115:75-83.

14. Abdel-Rahman O. Outcomes of surgery as part of the management of metastatic non-small-cell lung cancer: a Surveillance, Epidemiology and End Results database analysis. Cancer Invest 2018;36:238-45.

15. Okamoto J, Kubokura H, Usuda J. Factors determining the choice of surgical procedure in elderly patients with non-small cell lung cancer. Ann Thorac Cardiovasc Surg 2016;22:131-8.

16. Baldvinsson K, Oskarsdottir GN, Orrason AW, et al. Resection rate and operability of elderly patients with non-small cell lung cancer: nationwide study from 1991 to 2014. Interact Cardiovasc Thorac Surg 2017;24:733-9.

17. Surveillance, Epidemiology, and End Results (SEER) Program (www.seer.cancer.gov) SEER ${ }^{*}$ Stat Database: Incidence - SEER 9 Regs Research Data, Nov 2015 Sub (1973-2013) - Linked To County Attributes - Total U.S., 1969-2014 Counties, National Cancer Institute, DCCPS, Surveillance Research Program, Surveillance Systems Branch, released April 2016, based on the November 2015 submission.

18. Howlader N, Noone AM, Krapcho M, et al. Research Data (1973-2013), National Cancer Institute, DCCPS, Surveillance Research Program, Surveillance Systems Branch, Surveillance Epidemiology and End Results (SEER) Program (www.seer.cancer.gov). Released April 2016, based on the November 2015 submission.

19. Rosenbaum PR, Rubin DB. The central role of the propensity score in observational studies for causal effects. Biometrika 1983;70:41-55.

20. Rosenbaum PR, Rubin DB. Constructing a control group using multivariate matched sampling methods That incorporate the propensity score. Am Stat 1985;39:33-8.

21. Austin PC. An introduction to propensity score methods 
for reducing the effects of confounding in observational studies. Multivariate Behav Res 2011;46:399-424.

22. Koyi H, Hillerdal G, Kölbeck KG, et al. Non-small-cell lung cancer (NSCLC) in octogenarians in clinical practice. Anticancer Res 2016;36:5397-402.

23. Wang H, Yan L, Li C, et al. Surgical intervention may be a therapeutic option for NSCLC patients with AJCC stage IV: a large population-based study. Cancer Manag Res 2018;10:3219-26.

24. Kawano D, Takeo S, Katsura M, et al. Surgical treatment of stage IV non-small cell lung cancer. Interact Cardiovasc Thorac Surg 2012;14:167-70.

25. Kwint M, Walraven I, Burgers S, et al. Outcome of radical local treatment of non-small cell lung cancer patients with synchronous oligometastases. Lung Cancer 2017;112:134-9.

26. Shen H, Cao Y, Li X, et al. Surgical intervention improves survival for metastatic non-small cell lung cancer patients.

Cite this article as: Qiu C, Zhang S, Jin H, Zhang X, Ye J. Radical local treatment for stage IV non-small cell lung cancer in older adults: a propensity-score matched analysis of the SEER database. Transl Cancer Res 2020;9(9):5336-5349. doi: 10.21037/tcr-19-2796
Medicine (Baltimore) 2016;95:e3800.

27. Kasapoglu US, Arınç S, Gungor S, et al. Prognostic factors affecting survival in non-small cell lung carcinoma patients with malignant pleural effusions. Clin Respir J 2016;10:791-9.

28. Reck M. Pembrolizumab as first-line therapy for metastatic non-small-cell lung cancer. Immunotherapy 2018;10:93-105.

29. Reck M, Rodríguez-Abreu D, Robinson AG, et al. Pembrolizumab versus chemotherapy for PD-L1positive non-small-cell lung cancer. $\mathrm{N}$ Engl J Med 2016;375:1823-33.

30. Brahmer JR, Rodríguez-Abreu D, Robinson AG, et al. Health-related quality-of-life results for pembrolizumab versus chemotherapy in advanced, PD-L1-positive NSCLC (KEYNOTE-024): a multicentre, international, randomised, open-label phase 3 trial. Lancet Oncol 2017;18:1600-9. 